\title{
TOWARDS AN URBAN DEM GENERATION WITH SATELLITE SAR INTERFEROMETRY
}

\author{
Cristian Rossi $^{a}$, Thomas Fritz $^{a}$, Michael Eineder $^{a}$, Esra Erten $^{b}$, Xiao Xiang Zhu ${ }^{a, c}$ and Stefan Gernhardt ${ }^{c}$ \\ ${ }^{a}$ German Aerospace Center (DLR), Remote Sensing Technology Institute (IMF), Wessling, Germany $\downarrow$ cristian.rossi@dlr.de \\ ${ }^{b}$ Istanbul Technical University (ITU), Civil Engineering Faculty, Department of Geomatic Engineering, Maslak Istanbul, Turkey \\ ${ }^{c}$ Technical University Munich (TUM), Remote Sensing Technology (LMF), Munich, Germany
}

\section{Commission VII/2}

KEY WORDS: SAR, Interferometry, TanDEM-X, Urban Digital Elevation Models

\begin{abstract}
:
This paper analyzes the potentials offered by the TanDEM-X mission for the generation of urban DEMs. Operationally acquired urban Raw DEMs are validated using as reference a LiDAR DEM. The approach used for the validation is application-oriented as a subproduct is a derivation of a urban volume map. The mean buildings absolute height error is found to be below 5 meters. Neglecting the buildings geolocation errors, this value drops below 60 centimeters. Nevertheless, the operational municipal mapping is limited to the standard resolution of 12 meters. The limitation is attenuated with the use of high resolution spotlight data. High-resolution raw DEMs over Las Vegas and Berlin are first analyzed. Some modifications to the interferometric processing chain necessary to produce high quality DEMs are studied. The obtained results open a new perspective to the urban DEM generation with satellite SAR interferometry.
\end{abstract}

\section{INTRODUCTION}

The mapping of urban areas is a claimed task for many management applications, such as urban development monitoring, urban climate studies, and renewable energy surveys. Airborne data are widely used at this purpose for the generation of Digital Elevation Models (DEMs). LiDAR (Light Detection And Ranging) is a mature technology for obtaining DEMs in urbanized environment. Spaceborne Synthetic Aperture Radar (SAR) is on the contrary not largely exploited due essentially to the lack of suitable data. In fact, an essential prerequisite for a successful exploitation of SAR data finalized to urban reconstruction is a resolution capable to map the desired target. A second one, but not less important, is the absence of temporal decorrelation in between the two SAR acquisitions, or, in other words, an interferometric bistatic configuration. The TanDEM-X mission, started in June 2010, has as primary objective the generation of a global DEM following the high standard accuracy HRTI-3. The X-band sensors employed for the mission allow an accurate building mapping, hence accomplishing the first prerequisite. Additionally the mission constitutes the first bistatic SAR interferometer in space, letting to a faithful surface reconstruction free of atmospheric and temporal decorrelations. The acquisition mode used to achieve the HTRI-3 requirements is the stripmap one, with a final spatial DEM resolution of 12 meters. This resolution does not assure an accurate mapping of dense metropolitan areas, nevertheless semi-urban and industrial areas are well mapped as shown in Sec. 2 with a validation made using reference LiDAR data over Munich (Germany). The mission foresees however experimental spotlight acquisitions finalized to the generation of higher resolution DEMs following the HRTI-4 standards with a 6 meters spatial resolution. Considering this resolution, a denser urban mapping becomes then possible. First examples of urban experimental high-resolution DEM are shown over Las Vegas (USA) and Berlin (Germany) in Sec. 3. The processing chain adopted for the interferometric DEM generation, also called Raw DEM, is built in the Integrated TanDEM$\mathrm{X}$ Processor (ITP). Although the chain is highly optimized in a global scale, some modifications are necessary for the purposes of the paper. In particular, the spectral shift filtering, the coregistration, the interferogram generation and the phase unwrapping processing steps are redesigned towards an optimal configuration for the local municipal zone to map. The limits of an interferometric SAR city modelling are introduced in Sec. 4 as conclusive remark.

\section{OPERATIONAL URBAN RAW DEM VALIDATION}

The main goal of the TanDEM-X mission is the generation of a global DEM, thus including all the cities all over the world. The processing of Raw Data for the generation of the Raw DEM is performed by one single processor, the Integrated TanDEM$X$ Processor (ITP) (Breit et al., 2010), not described in this paper. ITP is optimally configured in every single processing stage to provide a Raw DEM close to the HRTI- 3 standards (relative point-to-point height accuracy of 2 meters for moderate terrain with slope inferior to $20^{\circ}$ in a $1^{\circ}$ by $1^{\circ}$ cell). In the processing facilities, for a standard 50 by 30 kilometers scene, the processing time required for the generation of the Raw DEM from the Raw Data is about 15 minutes. This high optimization follows the algorithms presented in (Fritz et al., 2011). Cities inside the scene are mapped at the standard posting of 12 meters. Even if the relative TanDEM-X height accuracy requirements are successfully accomplished (Rizzoli et al., 2012), they are too coarsely defined for urban zones. The cell used for the DEM height error specification is too large for an accurate analysis over a single city. A new strategy is then adopted for the validation of the absolute height of isolated buildings inside the DEM. It is based on the comparison between detected buildings in a reference LiDAR DEM and the TanDEM-X one. The input for the comparison is the Digital Buildings Model (DBM), derived through a difference between the DEM and its Digital Terrain Model (DTM). In principle a thresholding operation on the DBM would be sufficient to detect the buildings. In practice, due to the interferometric errors intrinsic to the urban DEM generation, such as geometric decorrelations or phase unwrapping issues (Rossi et al., 2011), a thresholding is not sufficient and a morphological operation, necessary to detect the underlying structures, is used. The simplest structuring element for the morphological operation is the 2-by2 square. By this way sharp rectangular edges representing the buildings - are extracted and noisy areas are discarded. Standard 


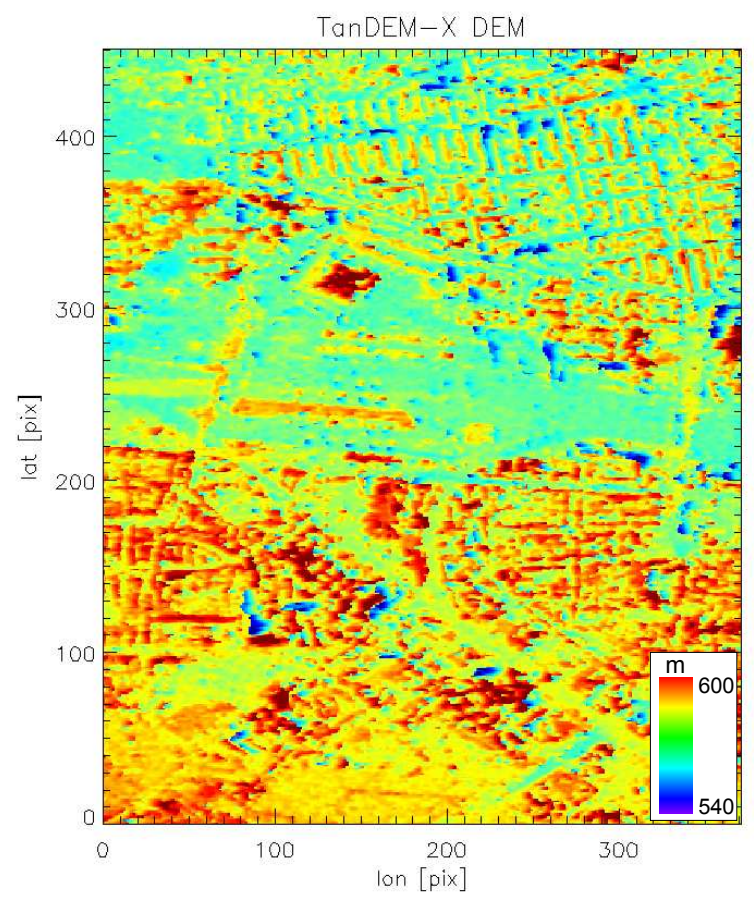

Figure 1: TanDEM-X operational raw DEM over the western part of Munich.

sequential operations of clumping and sieving are used respectively for connecting the gaps and removing isolated regions of the thresholded DBM. A segmentation operation is then needed to label the obtained buildings. A sub-product of this approach is a volume map:

$$
V_{N}=n_{N} \Delta_{l o n} \Delta_{l a t} \sum_{n} h_{n}
$$

where $V_{N}$ is the volume computed for the $N$ detected building, $n_{N}$ the number of pixels composing the building, $h_{n}$ the local heights and $\Delta$ the DEM pixel spacing. The chosen site is $\mathrm{Mu}-$ nich, with the operational Raw DEM acquired on the 31st October, 2010 used as test. The exploited Digital Terrain Model is the Digitales Gelaendemodell 25 (DGM25) from the Bayerische Vermessungsverwaltung. As the two models differ in resolution (DGM25 has 25 meters sampling), a resampling of the DGM25 on the TanDEM-X Raw DEM is firstly performed. The available LiDAR DEM covering the same area has a fine resolution of 0.5 meters, and is also resampled to the TanDEM one. In Fig. 1, the portion of the Raw DEM used for the validation is represented.

The results of the processing chain employed for the building detection in the TanDEM-X Raw DEM are shown in Fig. 2. The same chain is exploited for the LiDAR DEM. The difference between TanDEM-X and DGM25 is shown at the top left (mark (1)). The binary thresholding results are at the top right (mark (2)). The threshold is set to 5 meters. The LiDAR result is already satisfactory and a morphological operation could be avoided. On the contrary, the TanDEM-X result is noisier and a morphological operation to ensure the extraction of regularly shaped building is recommended. The results after the morphological stage are at the bottom left (mark (3)). The TanDEM result, even if not homogeneous like the LiDAR one, allows the detection of isolated buildings. The segmentation result, at the bottom right (mark
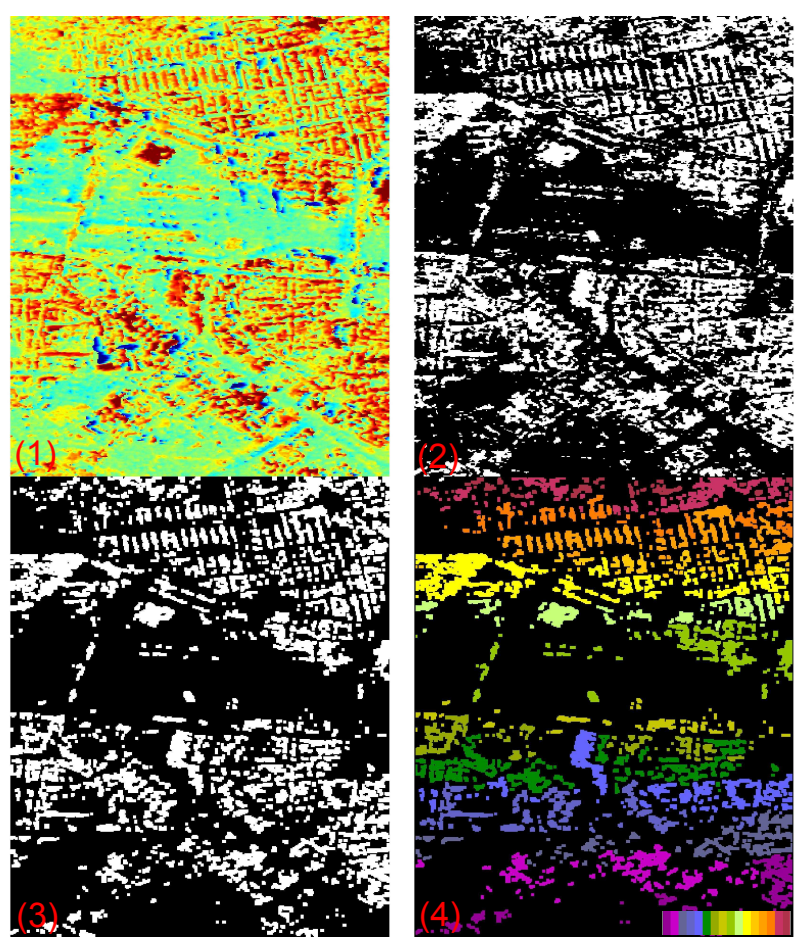

Figure 2: Processing result of the volume derivation chain for TanDEM-X Raw DEM. (1) DBM. (2) Binary Threshold. (3) Morphological operations. (4) Segmentation.

(4), color table at the bottom), numerates the detected structures. The TanDEM-X data overestimates the structures: using LiDAR data 403 structures are detected, whereas using TanDEM data the detection count is 437 (an increase of about 8\%). Looking into detail the errors, the wrongly detected buildings are in fact phase unwrapping errors (as the region at the upper left of the scene, which is a flourishing park) or a mixture of geometrical decorrelations (as the district at the bottom right of the scene, composed by small houses). A good accordance in the segmentation is achieved at the upper right district, composed by regularly located buildings. There is a general accordance for large buildings. The LiDAR segmentation, represented through the volume map, is shown in Fig. 3.

The two segmentation results are exploited for the computation of the buildings height difference. The accuracy of the operational stripmap TanDEM-X urban DEM is tested in two configurations. The accuracy of the mean height of the buildings considering the LiDAR segmentation is firstly tested. The LiDAR segmentation may differ with the TanDEM one at the building borders due to layover and shadow (Fig. 2 (4) and Fig.3) decreasing the accuracy. Secondarily, the common building areas, considering the LiDAR segmentation, is tested (Fig. 4). The results are in Tab. 1. Considering the LiDAR segmentation, the mean difference over all the detected buildings is 4.5 meters with a standard deviation in the same order size. The relatively high mean/standard deviation is caused by a dozen of buildings for which the TanDEM-X building geolocalization is not fitting the LiDAR one, so introducing also ground components in the measure. Just considering the common covered area the mean decreases to about 0.6 meters.

\section{EXPERIMENTAL URBAN RAW DEM GENERATION}

The high resolution allowed by the X-band sensors of the TanDEM$\mathrm{X}$ mission dramatically increases the potentials of a urban DEM 


\begin{tabular}{|c||c|c|c|c|}
\hline & Mean Difference [m] & STD Difference [m] & Mean RMSE [m] & STD RMSE [m] \\
\hline \hline LiDAR Segmentation & 4.536 & 4.334 & 8.205 & 4.249 \\
\hline Common Segmentation & 0.589 & 3.743 & 4.824 & 4.028 \\
\hline
\end{tabular}

Table 1: Operational Urban TanDEM-X Raw DEM Accuracy

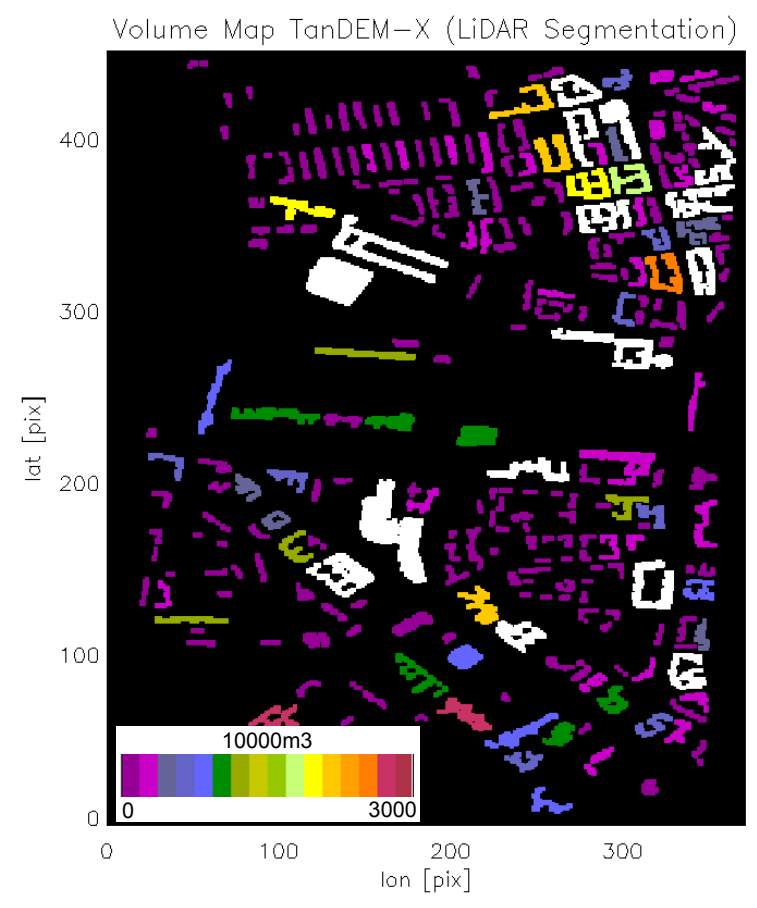

Figure 3: Volume map derived from TanDEM-X data using the LiDAR segmentation result.

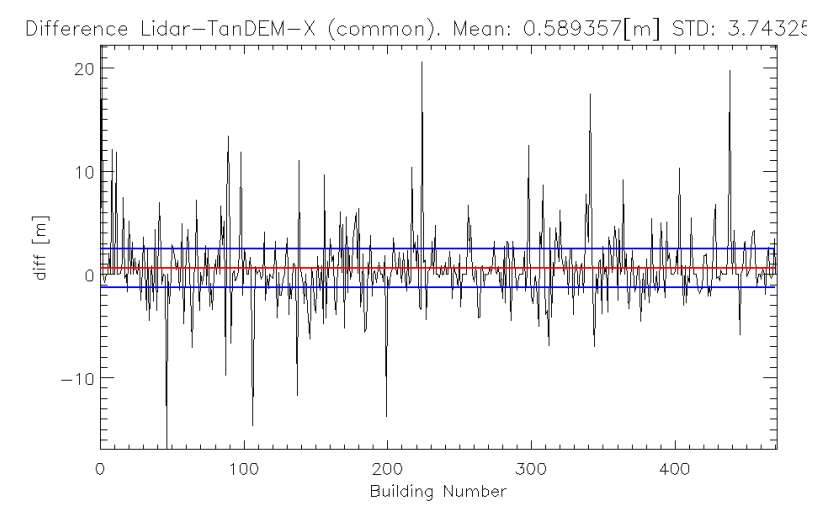

Figure 4: Buildings mean height difference between LiDAR and TanDEM-X using for LiDAR segmentation. The red and blue lines represent respectively the measured mean and the standard deviation.

generation. The operational interferometric processing could be modified towards optimal solutions for the processing of metropolitan regions. The processing chain involves sequentially the spectral filtering, the coregistration, the interferogram generation and its multilooking, the phase unwrapping and the geocoding (Rossi et al., 2012). Besides the range spectral filtering and the phase unwrapping, which can be switched off depending on the scene configuration, this paper analyzes modifications to the coregistration and multilooking stages. The modification to the geocoding stage are here not yet studied.

\subsection{Spectral Shift and Phase Unwrapping Stages}

The spectral shift stage could be switched off in case of pure urban areas. The statistical base which justifies it, distributed scattering, is generally not valid for municipal zones. Generally, the geometrical configuration of the TanDEM-X mission is built to obtain a small gain from the filtering, of about the 3\%-5\%. This processing step can be thus enabled to obtain a small gain for mixed scene configuration (rural, urban).

The phase unwrapping algorithm exploited in ITP is the Minimum Cost Flow (MCF). If the overall height variations of the scene are smaller than the height of ambiguity it could be in principle switched off. Considering the mission planning it is anyhow always turned on, as the first year height of ambiguity is around 45 meters and the second one around 35 meters. Scene height variations not overcoming these boundaries are quite uncommon.

\subsection{Coregistration Stage}

The algorithm exploited in ITP is already optimized, with misalignments well below the pixel (Yague Martinez et al., 2010). Nevertheless, it can be configured through the coregistration window size and distance. In the HR spotlight case, the window size is set to about 35 meters in azimuth direction and to 19 meters in the range one. The distance between windows is respectively 70 and 30 meters. Tradeoffs between window size and desired accuracy were already predicted for the coherent case (Bamler, 2000). Due to different statistics they are however not valid for urban scenarios. In the urban case, large windows or large distances may include different building with different heights, creating a coregistration mismatch and a loss of coherence. For a standard TanDEM-X scenario the loss of coherence can be quantified with geometrical calculations. The result for different height discrepancies in a coregistration window cell is in Fig. 5. Due to the relatively small baselines of the helix formation of TanDEM-X the loss is unimportant (a coherence of about 0.05 for a height discrepancy of 100 meters). The ITP coregistration approach can be thus considered already optimized. A small suggestion would be the reduction of the window distance by a factor of 2 . It has been in fact empirically proven that the reduction of the distance reduces the number of phase unwrapping residues by a small amount.

\subsection{Interferogram Generation Stage}

The highly optimized moving average window employed to reduce the phase noise in the ITP multilook stage can be optimized for urban modelling purposes. In particular, adaptive algorithms making use of amplitude statistics to fuse pixels with the same features are here analyzed. The algorithm in (Vasile et al., 2004), connecting pixels with a region growing technique, and the one in (Deledalle et al., 2011), connecting also not consecutive pixels inside a search window, are tested. The need to employ adaptive methods is clear looking at Fig. 7 and Fig. 8, portions of the DEM for an high resolution spotlight acquisition over Las Vegas acquired on the 25th September, 2011. The interferometric phase in Fig. 6 is processed to obtain a mean theoretical resolution of 3.65 meters. The NL-InSAR algorithm (Deledalle et al., 2011) is used for the multilooking and the coherence estimation. The 


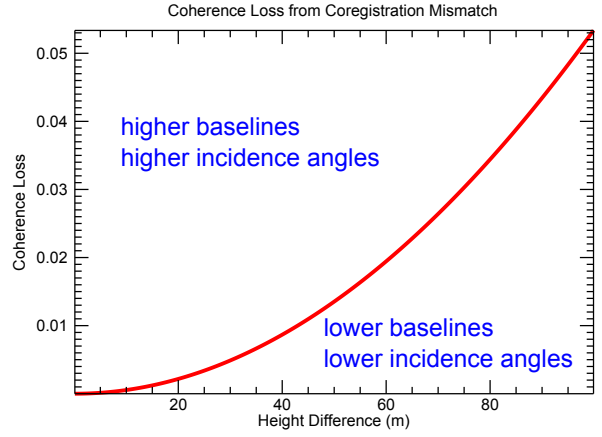

Figure 5: Coherence loss due to a coregistration mismatch in a typical TanDEM-X scenario.

phase noise mitigation is evident comparing the two DEM portions. The height variance of the flat roof of the four buildings in the middle of the portion is 4.72 meters for the operational case and 0.68 meters for the experimental one. The improvement is great also for the phase unwrapping stage, necessary in this case due to the topography visible in Fig. 6. The combined use of the adaptively multilooked phase and the refined coherence in creases dramatically the phase unwrapping quality ratio $q_{r}$. This ratio expresses the percentage of the scene not affected by phase unwrapping errors (Rossi et al., 2012). Whereas in the operational scenario $q_{r}=76 \%$, meaning that about one quarter of the scene is affected by phase unwrapping errors, in the experimental one $q_{r}=99.9 \%$, meaning that the adaptive processing totally solved the phase unwrapping issue. The false heights in the operational scenario are caused by the very low coherent road at the center-left of the scene (Fig. 9). The interferometric phase of the road is highly noisy, creating several branch cuts. In the phase unwrapping stage, the two sides of the road remains unconnected, creating an hight discrepancy between them of one phase cycles ( 35 meters). This problem is solved employing the adaptive multilooking technique, as shown from the radargrammetric control maps (Rossi et al., 2012) in Fig. 10 and 11. Thus, the combined use of the enhanced phase and coherence in the employment and cost computation of the MCF algorithm provides a better result in the reduction of false heights generated by the phase unwrapping stage.

The noise mitigation algorithms can be used also to reduce the theoretical DEM resolution. An example of Raw DEM over Berlin, processed with the region growing algorithm (Vasile et al., 2004), with a theoretical resolution of 2 meters, is shown in Fig. 12. The relative flatness of the scene allows the identification of a great amount of details from this two-dimensional view. A portion of the DEM over the city center is in Fig. 13. The height jumps over the river are caused by the adaptive multilooking algorithm. The height depressions are mostly shadow areas in the SAR image. Overall, buildings are well represented even at the imposed small resolution.

\section{CONCLUSIONS AND FUTURE WORK}

This paper showed the potentials of TanDEM-X in the mapping of urban areas, globally acquired by the mission. Even if the operational Raw DEM has a coarse 12 meters resolution for a dense urban mapping, it has been showed through an application oriented algorithm how the mean absolute height accuracy

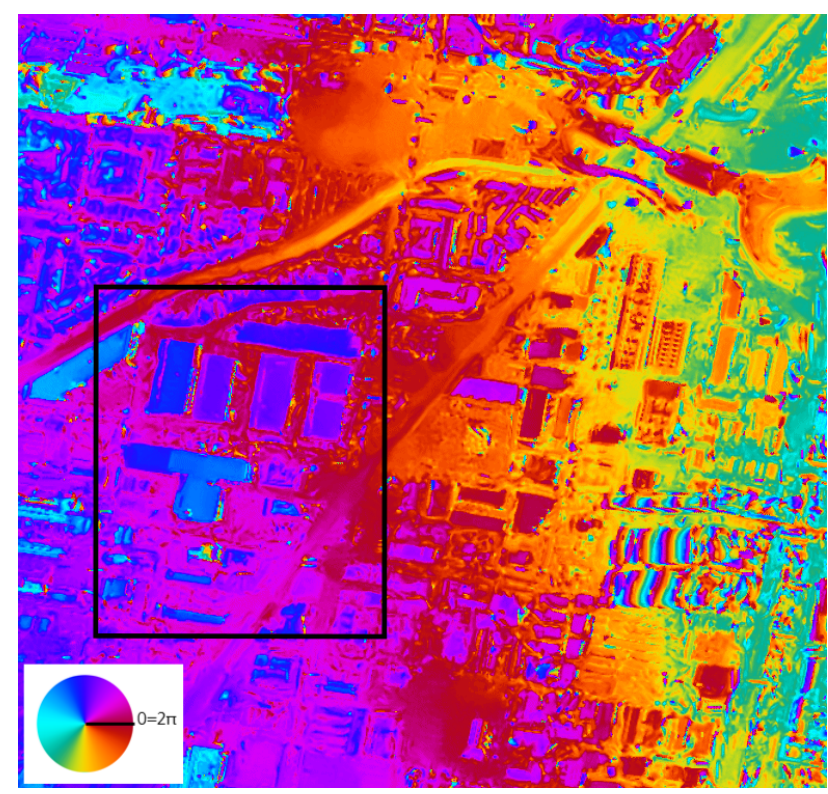

Figure 6: Interferometric phase of a portion of an acquisition over Las Vegas. In the black box the buildings under analysis.

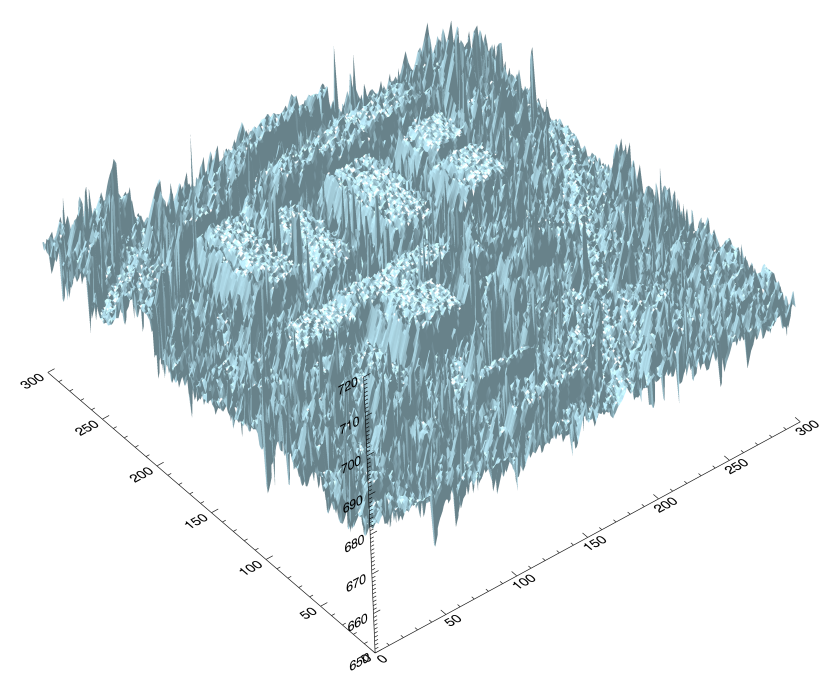

Figure 7: Three dimensional visualization of the DEM over Las Vegas for an industrial area generated using operational algorithms.

of detected buildings in the Raw DEM is below 5 meters. Not considering the segmentation errors due to the geometrical decorrelations, the mean error drops below the meter and the standard deviation is kept below four meters. The building geolocalization accuracy has planned to be studied with the help of the Permanent Scatterer Interferometry high resolution technique. In the paper first examples of experimental high resolution urban Raw DEMs have been provided. The quality of the phase has been enhanced using the SAR amplitude statistics through adaptive multilooking techniques. The enhanced phase, together with the enhanced coherence, helped the phase unwrapping stage. This enhancement allowed the generation of fine Raw DEMs having a theoretical resolution of just two meters. The absolute height and the geolocation validation of very high resolution DEMs are planned to be performed. Nonetheless, this paper didn't analyze the limitations of the building reconstruction from a single aspect TanDEM acquisition. The greatest limitation to a urban three dimensional re- 


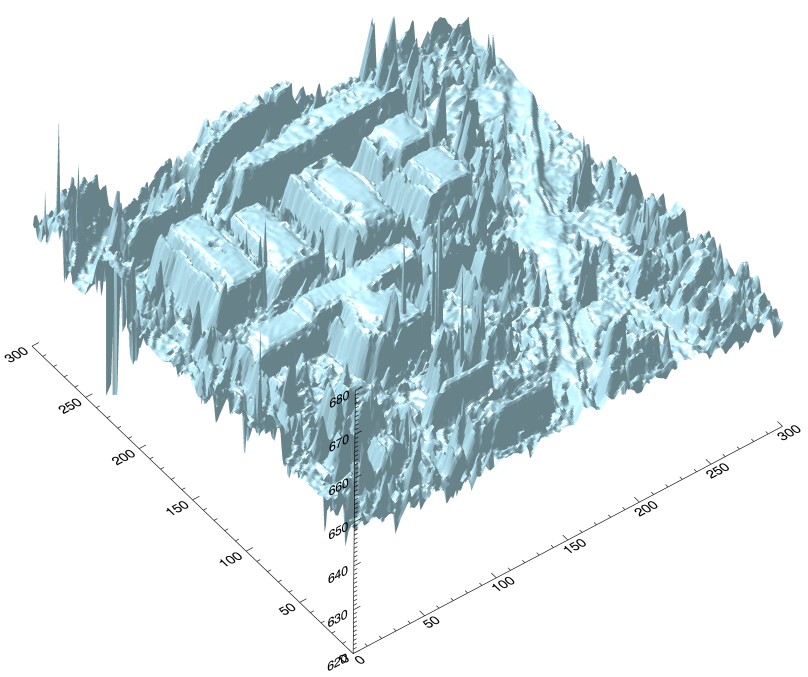

Figure 8: Three dimensional visualization of the DEM over Las Vegas for an industrial area experimentally generated using the NL-InSAR technique.

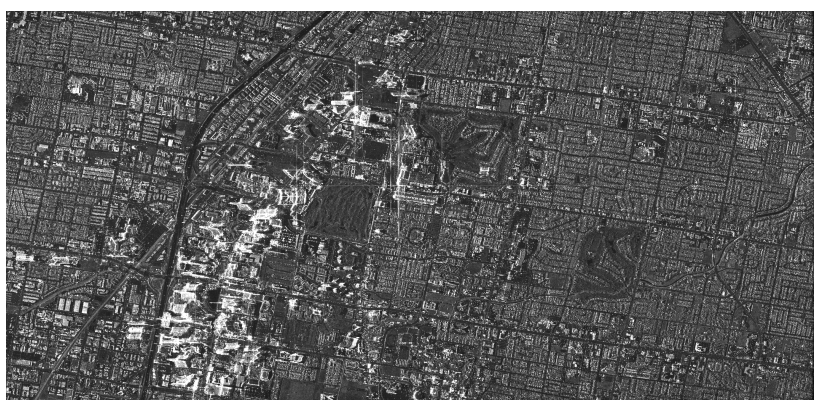

Figure 9: Master channel amplitude of the high resolution spotlight acquisition acquired over Las Vegas on the 25th September, 2011.

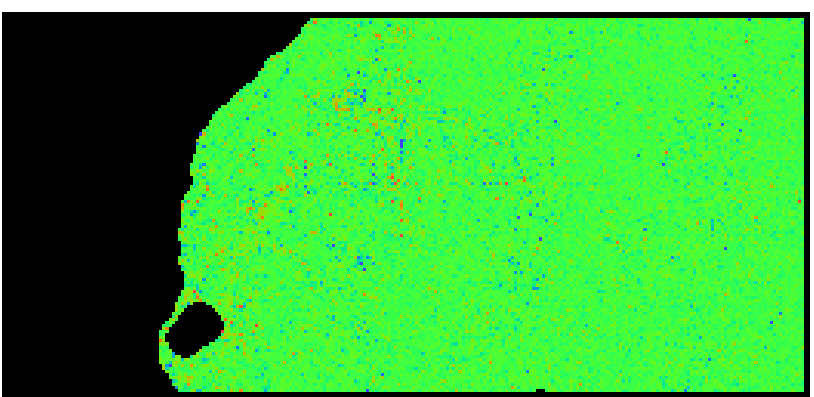

Figure 10: Radargrammetric control map for the Raw DEM operationally processed. The black area corresponds to the phase unwrapping error, spanning the $24 \%$ of the scene.

construction takes its origin from the side looking geometry of the radar sensors. Issues like layover and shadow remain unsolved in the single aspect case. Their effect in the DEM are a height slope and a wrong geolocation for the layover case and a random height for the shadow one. The use of ascending and descending tracks helps to resolve the shadowing effect, while the use of tomographic techniques could resolve the layover case. Thus, only the use of multi aspect data or a stack of acquisitions would help the three dimensional reconstruction. In any case, the urban Raw DEMs generated by the TanDEM-X mission allow the mapping of single buildings with a great amount of details, being then

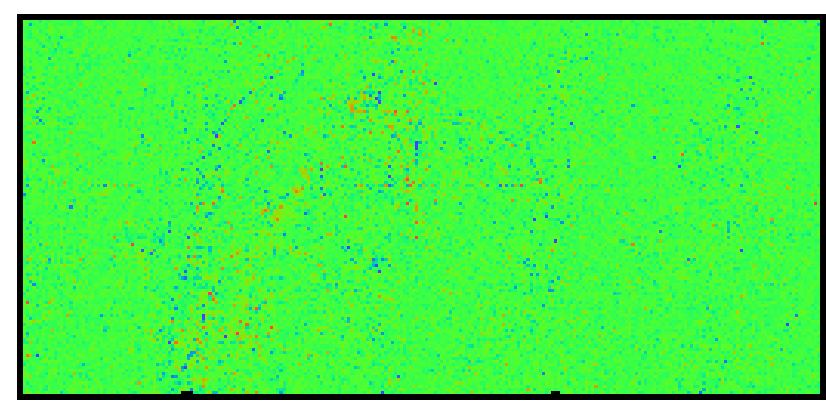

Figure 11: Radargrammetric control map for the Raw DEM experimentally processed.

useful in all the applications requiring a municipal mapping.

\section{ACKNOWLEDGEMENTS}

The TanDEM-X project is partly funded by the German Federal Ministry for Economics and Technology (Foerderkennzeichen 50 EE 1035). This work is part of the Tomopolis project, founded by the Deutsches Zentrum fuer Luft und Raumfahrt e.V. RaumfahrtAgentur.

\section{REFERENCES}

Bamler, R.; Interferometric stereo radargrammetry: absolute height determination from ERS-ENVISAT interferograms, Geoscience and Remote Sensing Symposium (IGARSS), 2000 IEEE International, vol.2, pp.742-745

Breit, H.; Fritz, T.; Balss, U.; Niedermeier, A.; Eineder, M.; Yague-Martinez, N.; Rossi, C.; Processing of Bistatic TanDEM$\mathrm{X}$ data, Geoscience and Remote Sensing Symposium (IGARSS), 2010 IEEE International, pp.2640-2643, Honolulu, 25-30 July 2010

Deledalle, C.-A.; Denis, L.; Tupin, F.; NL-InSAR: Nonlocal Interferogram Estimation, Geoscience and Remote Sensing, IEEE Transactions on, vol.49, no.4, pp.1441-1452, April 2011

Fritz, T.; Rossi, C.; Yague-Martinez, N.; Rodriguez-Gonzalez F.; Lachaise, M.; Breit, H.; Interferometric Processing of TanDEMX Data, Geoscience and Remote Sensing Symposium (IGARSS), 2011 IEEE International, Vancouver, July 24-29, 2011

Rizzoli, P,; Brautigam, B; Krauss, T; Martone, M., Krieger, G.; Relative Height Error Analysis of TanDEM-X Elevation Data, ISPRS J. Photogramm. Remote Sens., under review, 2012

Rossi, C.; Fritz, T.; Breit, H.; Eineder, M.; First Urban TanDEM-X RawDEM Analysis, Joint Urban Remote Sensing Event (JURSE), pp.65-68, 11-13 April 2011

Rossi, C.; Rodriguez Gonzalez, F.; Fritz, T.; Yague Martinez N.; Eineder, M.; Calibrated TanDEM-X Raw DEM Generation, ISPRS J. Photogramm. Remote Sens., under review, 2012

Vasile, G.; Trouv, E.; Ciuc, M. and Buzuloiu, V.; General adaptive-neighborhood technique for improving synthetic aperture radar interferometric coherence estimation, J. Opt. Soc. Am. A 21, 1455-1464, 2004

Yague-Martinez, N.; Eineder, M.; Brcic, R.; Breit, H.; Fritz, T.; TanDEM-X Mission: SAR Image Coregistration Aspects, Synthetic Aperture Radar (EUSAR), 2010 8th European Conference on, Aachen, 7-10 June 2010 


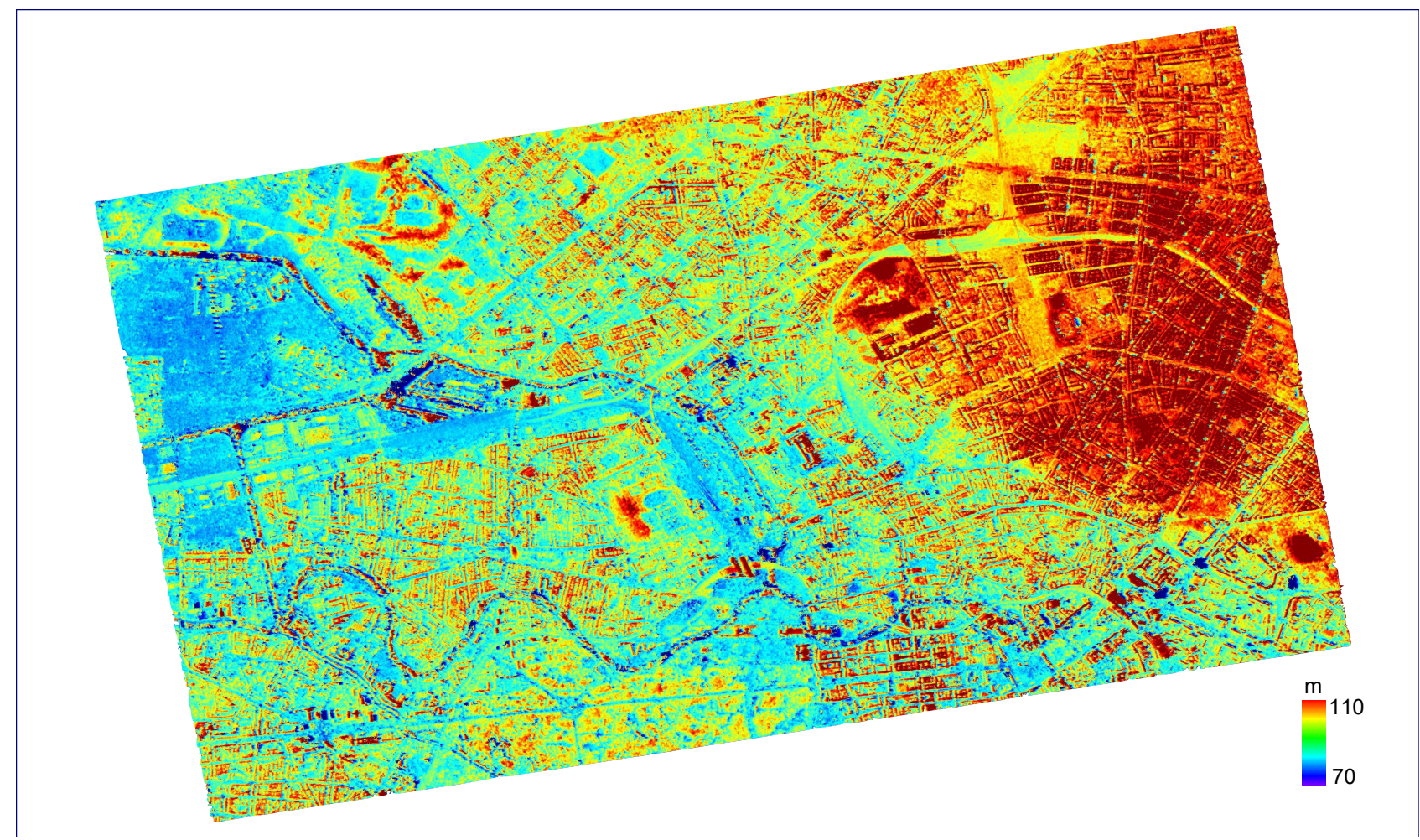

Figure 12: Experimental 2 meters resolution Raw DEM over Berlin, generated from a TanDEM-X acquisition on the 04th January, 2012.

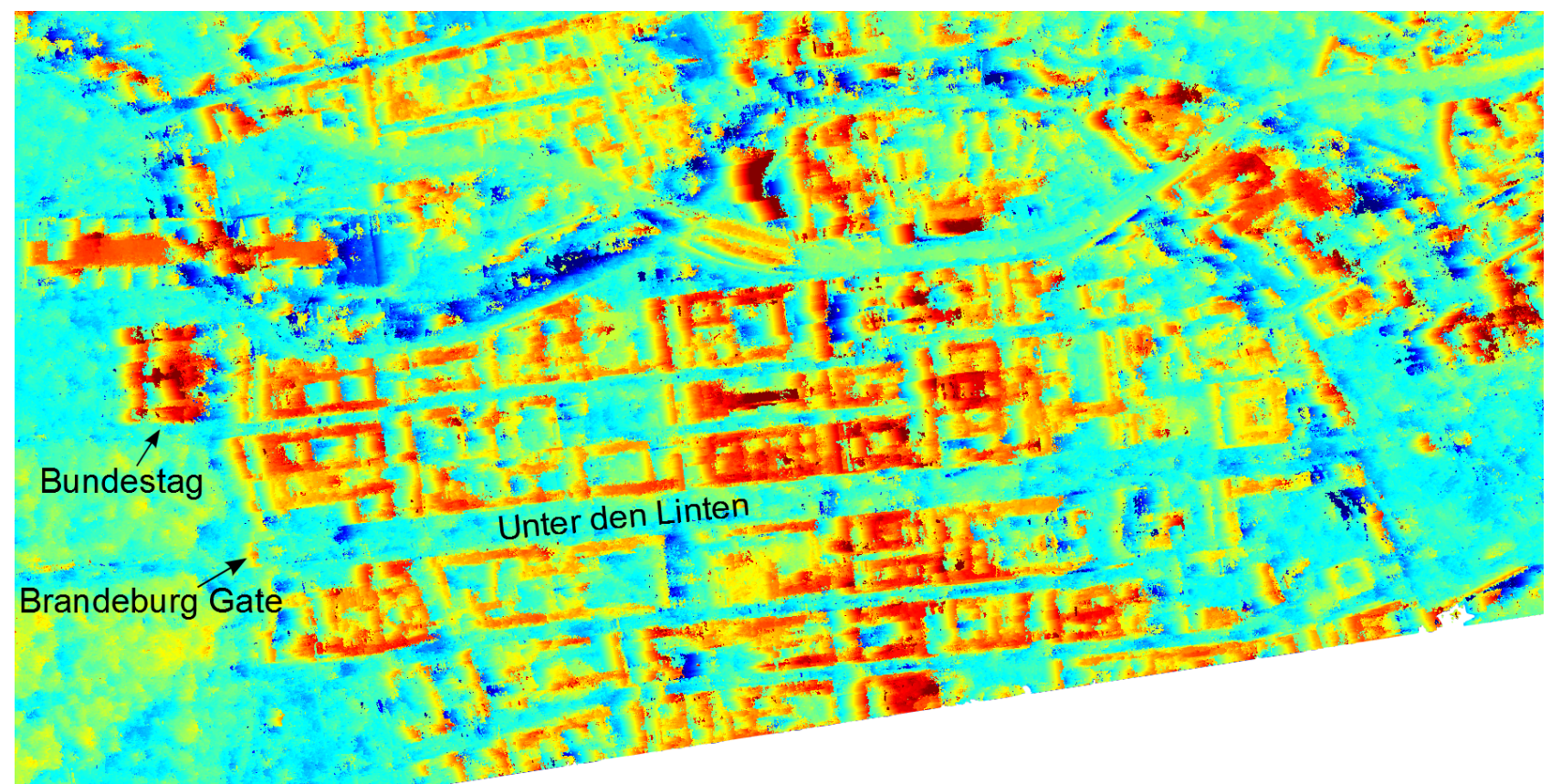

Figure 13: Portion of the experimental Raw DEM over the city center. 